\title{
Hypermethioninemia encephalopathy due to adenosine kinase deficiency
}

INSERM

\section{Source}

INSERM. (1999). Orphanet: an online rare disease and orphan drug data base.

Hypermethioninemia encephalopathy due to adenosine kinase deficiency.

ORPHA:289290

Hypermethioninemia encephalopathy due to adenosine kinase deficiency is a rare inborn error of metabolism disorder characterized by persistent hypermethioninemia with increased levels of S-adenosylmethionine and S-adenosylhomocysteine which manifests with encephalopathy, severe global developmental delay, mild to severe liver dysfunction, hypotonia and facial dysmorphism (most significant is frontal bossing, macrocephaly, hypertelorism and depressed nasal bridge). Epileptic seizures, hypoglycemia and/or cardiac defects (pulmonary stenosis, atrial and/or ventricular septal defect, coarctation of the aorta) may be associated. Clinical picture may range from neurological symptoms only to multi-organ involvement. 